\title{
Are 5-7 Days of Folic Acid Supplementation Necessary prior to Pemetrexed? Observations from a Case Series
}

\author{
Benjamin R. Griffin ${ }^{a} \quad$ Elisabeth K. Stephens ${ }^{b, c} \quad$ Aminah Jato $^{b, c}$ \\ ${ }^{a}$ Department of Medicine, Mayo Clinic, ${ }^{b}$ Cancer Education Program, Mayo Clinic \\ Cancer Center, and ${ }^{C}$ Department of Oncology, Mayo Clinic, Rochester, Minn., USA
}

\section{Key Words}

Folic acid · Pemetrexed · Adverse events · Premedication

\begin{abstract}
Objective: Current regulatory guidelines advise 5-7 days of folic acid supplementation prior to pemetrexed. Although taking folic acid during and after pemetrexed therapy is important, it remains unclear whether premedication is truly necessary, particularly as it can be inconvenient for patients, can contribute to their anxiety by delaying chemotherapy, and can create challenges in scheduling chemotherapy. Methods and Results: We retrospectively sought to identify and evaluate outcomes among non-small cell lung cancer patients who received less than the advised folic acid premedication. Only 8 patients were identified. However, upon critical examination of first-cycle chemotherapy outcomes, we observed no major adverse events with a shortened course of folic acid premedication. Conclusion: In the very rare circumstance where urgent therapy is warranted, a healthcare provider can lookto this small case series and find modest precedent for the safe administration of pemetrexed in the absence of a full week of folic acid premedication.
\end{abstract}

\section{Introduction}

The Food and Drug Administration's patient package insert for pemetrexed (Alimta) reads as follows [1]: 'You must start taking 350-1,000 mcg of folic acid every day for at least 5 days out of the 7 days before your first dose of Alimta'. 
This advice is a direct outgrowth of earlier studies showing that in half of the patients, pemetrexed resulted in the development of grade 3/4 myelosuppression, mucositis, diarrhea, and other major adverse events with mortality rates that reached 4\% $[2,3]$. Understanding this agent's mechanism of action, acknowledging data that suggest a salutary effect of folic acid supplementation in pemetrexed-exposed animals and revisiting parallel data which show a better side effect profile with folate supplementation with methotrexate (another antifolate agent) led investigators to test this vitamin as a means to attenuate pemetrexed's formerly unacceptable adverse event profile [4]. Indeed, Bunn et al. [5] described that folic acid supplementation resulted in a drop in grade 4 neutropenia from 32 to $3 \%$ and led to a decline in grade $3 / 4$ mucositis from 5 to $1.3 \%$ as well as a plummeting of grade- 5 events from 5 to $0 \%$. Premedication with folic acid for 5-7 days, continuation throughout the pemetrexed chemotherapy cycle, and maintenance of this vitamin for 3 weeks after discontinuation of pemetrexed dramatically reduced toxicity.

However, the importance of initiating folic acid for 5-7 days prior to pemetrexed seems uncertain. The resultant 1 -week chemotherapy delay might only rarely affect clinical outcomes but is inconvenient for patients, can contribute to patients' anxiety, and can create challenges in scheduling chemotherapy. Moreover, because adverse events typically begin approximately 1 week after chemotherapy administration and because folic acid is a watersoluble vitamin, a full week of premedication may be unnecessary. Hence, this study was undertaken to explore the very practical question of whether 5-7 days of folic acid supplementation prior to pemetrexed is truly necessary.

\section{Methods}

The Mayo Clinic Institutional Review Board reviewed and approved this study. The study team then contacted the Mayo Clinic Tumor Registry to acquire a list of all metastatic non-small cell lung cancer patients who had been diagnosed during or after 2004 (to coincide with the Food and Drug Administration approval of this drug for lung cancer) and who had received their chemotherapy at our institution.

The medical records of these patients were reviewed by a team member (B.R.G.) to identify those who had received pemetrexed and, more specifically, those who had received this drug without a full week of folic acid premedication.

The medical records of this latter group of patients were then reviewed in depth by all members of the study team to capture patient demographics, other concomitant chemotherapy agents, multivitamin use, folic acid use after pemetrexed administration, and adverse events that occurred during the first cycle of chemotherapy.

\section{Results}

Demographics

From the initial group of over 200 non-small cell lung cancer patients, 81 had received pemetrexed; 8 of these did not receive a full week of folic acid prior to the initiation of chemotherapy. The mean age in this latter small subgroup was 67 years (range 50-77), and gender was evenly represented. 
Griffin et al.: Are 5-7 Days of Folic Acid Supplementation Necessary prior to

Pemetrexed? Observations from a Case Series

\section{Reasons for Shortened Folic Acid Premedication}

In 2 patients, folic acid premedication was shortened because of an urgency to start chemotherapy, in 2 it was reduced for logistical reasons, and in the remaining 4 patients, the reason for the reduction was unclear.

Folic Acid, Multivitamins, and Other Chemotherapy Agents

Two patients had been taking a multivitamin well prior to pemetrexed, but the dose of folic acid within the multivitamin remains unknown. All other patients were eventually prescribed folic acid concurrently with pemetrexed and appeared adherent to vitamin supplementation. All patients received more than one antineoplastic agent (see table 1).

\section{Adverse Events}

Only 1 patient was hospitalized (patient 1 in table 1) after her first course of pemetrexed. This patient had been hospitalized earlier for shortness of breath from a pulmonary embolus and was readmitted with fevers and pneumonia. She improved after the initiation of antibiotics and with continued anticoagulation. This patient had no myelosuppression or other major pemetrexed-related toxicity.

\section{Discussion}

This case series illustrates that the majority of patients at our institution receives 5-7 days of folic acid prior to pemetrexed. Yet, the 8 patients who did not receive a full week of folic acid premedication prompt the question of whether delaying chemotherapy for this purpose is necessary. In this series, only 1 patient was hospitalized but, in the absence of neutropenia, it is unclear whether this hospitalization could truly be attributable to chemotherapy. Furthermore, the other patients described here did well after their first cycle of chemotherapy, without grade 3 or worse adverse events. In view of the importance of expediting medical therapy, attempting to decrease cancer patients' anxiety from having to delay chemotherapy, and allowing greater flexibility in scheduling chemotherapy, these results suggest that further study of folic acid premedication is indicated.

Of note, our findings are preliminary for at least two reasons. First, the United States' food supply has been fortified with folic acid since 1997 - approximately the same time that the early pemetrexed studies without folic acid were being completed and the need for folic acid supplementation with pemetrexed was beginning to be examined and tested [6]. Thus, whereas in the United States the need for pretreatment with folic acid might be avoided, such might not be the case in other parts of the world where folic acid fortification of the food supply is nonexistent. Second, this small case series is just that: a small case series, thus making it impossible to draw large, sweeping conclusions or to provide even tentative, practice-changing suggestions. Based on the preliminary observations described here, we can only conclude that further study of folic acid premedication with pemetrexed is indicated. In the very rare circumstance where urgent therapy is warranted, a healthcare provider can look to this small case series and find modest precedent for the safe administration of pemetrexed in the absence of a full week of folic acid premedication.

\section{Acknowledgment}

This work was funded by K24CA131099. 


\section{Case Reports in Oncology}

\begin{tabular}{l|l}
\hline Case Rep Oncol 2013;6:339-342 \\
\hline DOI: $10.1159 / 000353573$ & $\begin{array}{l}\text { C 2013 S. Karger AG, Basel } \\
\text { www.karger.com/cro }\end{array}$ \\
\hline
\end{tabular}

Griffin et al.: Are 5-7 Days of Folic Acid Supplementation Necessary prior to Pemetrexed? Observations from a Case Series

\section{References}

1 http://www.accessdata.fda.gov/drugsatfda_docs/label/2004/021462ppilbl.pdf (accessed February 21, 2013).

-2 John W, Picus J, Blanke CD, et al: Activity of multitargeted antifolate (pemetrexed disodium, LY231514) in patients with advanced colorectal carcinoma: results from a phase II study. Cancer 2000;88:1807-1813.

-3 Pivot X, Raymond E, Laguerre B, et al: Pemetrexed disodium in recurrent locally advanced or metastatic squamous cell carcinoma of the head and neck. Br J Cancer 2001;85:649-655.

4 Worzalla JF, Shih C, Schultz RM: Role of folic acid in modulating the toxicity and efficacy of the multitargeted antifolate, LY231514. Anticancer Res 1998;18:3235-3239.

5 Bunn P, Paoletti P, Niyikiza C, et al: Vitamin B12 and folate reduce toxicity of Alimta (pemetrexed disodium, LY231514, MTA), a novel antifolate/antimetabolite (abstract 300). ASCO Annual Meeting, San Francisco, 2001.

6 Kim Y-I: Will mandatory folic acid fortification prevent or promote cancer? Am J Clin Nutr 2004;80:11231128.

Table 1. Folic acid, multivitamins, pemetrexed, and other chemotherapy agents

\begin{tabular}{|c|c|c|c|c|c|c|}
\hline $\begin{array}{l}\text { Patient } \\
\text { number }\end{array}$ & $\begin{array}{l}\text { Days of } \\
\text { folic acid } \\
\text { premedication }\end{array}$ & $\begin{array}{l}\text { Dose of } \\
\text { folic acid }\end{array}$ & $\begin{array}{l}\text { Daily } \\
\text { multivita- } \\
\text { min }\end{array}$ & $\begin{array}{l}\text { Dose of } \\
\text { pemetrexed } \\
\mathrm{mg} / \mathrm{m}^{2}\end{array}$ & $\begin{array}{l}\text { Other concomita } \\
\text { chemotherapy } \\
\text { agents }\end{array}$ & t Adverse events \\
\hline 1 & 2 & $1 \mathrm{mg} /$ day & no & 500 & carboplatin & $\begin{array}{l}\text { hospitalized with } \\
\text { pneumonia and pulmonary } \\
\text { embolus }\end{array}$ \\
\hline 2 & 2 & $1 \mathrm{mg} /$ day & no & 400 & carboplatin & no \\
\hline 3 & 0 & unknown & no & 500 & bevacizumab & no \\
\hline 4 & 2 & unknown & yes & 500 & carboplatin & low-grade stomatitis \\
\hline 5 & 4 & $400 \mathrm{mcg} /$ day & no & 500 & $\begin{array}{l}\text { carboplatin, } \\
\text { bevacizumab }\end{array}$ & no \\
\hline 6 & 5 & $400 \mathrm{mcg} /$ day & yes & 500 & carboplatin & no \\
\hline 7 & 2 & $400 \mathrm{mcg} /$ day & no & 500 & carboplatin & no \\
\hline 8 & 2 & unknown & no & 400 & carboplatin & no \\
\hline
\end{tabular}

